\title{
Extension of the Pirogov-Sinai Theory to a Class of Quasiperiodic Interactions ${ }^{\star}$
}

\author{
F. Koukiou ${ }^{1}$, D. Petritis ${ }^{1}$ and M. Zahradník ${ }^{2}$ \\ ${ }^{1}$ Institut de Physique Théorique, Université de Lausanne, CH-1015 Lausanne, Switzerland \\ ${ }^{2}$ Department of Mathematics, Charles University, Sokolovská 83, CS-18600 Prague, Czechoslovakia
}

\begin{abstract}
We extend the Pirogov-Sinai theory in such a manner that it applies to a large class of models with small quasiperiodic interactions as perturbations of periodic ones. We find general diophantine conditions on the frequency module of the quasiperiodic interactions and derivability conditions on the interaction potentials. These conditions allow to prove that the low temperature phase diagram is a homeomorphic deformation of the phase diagram at zero temperature.
\end{abstract}

\section{Introduction and Main Results}

The standard Pirogov-Sinai theory studies the low temperature phase diagram for discrete spin systems on a lattice described by hamiltonians with finite range of interactions which are translational invariant or periodic. Namely, for hamiltonians having a finite $(m+1)$ number of constant or periodic ground states and satisfying the Peierls condition, the Pirogov-Sinai theory asserts that the topological structure of the phase diagram at sufficiently low temperature is the same as that of the diagram at zero temperature $[15]$ (see $[18,19]$ for pedagogical expositions).

In order to study the structure of the phase diagram, the original hamiltonian $H_{0}$ which is assumed to have exactly $m+1$ ground states, is perturbed by small additional terms associated to a vector of coupling constants $\xi=\left(\xi_{1}, \ldots, \xi_{m}\right) \in \mathbb{R}^{m}$. For example one could consider

$$
H_{\xi}=H_{0}+\sum_{i=1}^{m} \xi_{i} H_{i}
$$

(non-linear dependence of $H_{\xi}$ is also possible). One requires the perturbations $H_{i}$ to remove the degeneracy of the hamiltonian $H_{0}$. The phase diagram at zero temperature is obtained by minimizing $H_{\xi}$ in the space of the parameters $\xi$. In general, this phase diagram has the following topological structure: there is a point

\footnotetext{
* Work partially supported by Swiss National Science Foundation
} 
of coexistence of $m+1$ ground states in the space of the parameters (which is isomorphic to $\mathbb{R}^{m}$ ); from this point emanate: $m+1$ half-lines on which $m$ ground states coexist, two dimensional linear surfaces having pairs of the previous lines as boundaries on which $m-1$ ground states coexist, etc. The theorem of [15] states that the phase diagram at sufficiently low temperature is obtained as a homeomorphic transformation of the diagram at zero temperature, hence, its topological structure is completely preserved.

In the last years many developments and generalizations were done on the original Pirogov-Sinai theory. Without being exhaustive let us mention a few of them: a generalization to $P(\phi)_{2}$ models in field theory [9], generalization to continuous spin systems [6], extension to a class of interactions having an infinite number of ground states [3], to rapidly decaying infinite range interactions (excluding Coulomb potentials) [14]. In [20] the completeness of the phase diagram is shown.

Some alternative versions of Pirogov--Sinai theory also appeared. In [20] the technical intermediate steps are treated in a different manner avoiding the introduction of a parametric polymer model. In [7] the rigorous renormalization group approach was used; moreover this powerful generalization extends the Pirogov-Sinai theory to cover the case of complex interactions. Analyticity of the Pirogov-Sinai diagram was proved in $[1,21]$.

As applications of the theory one can mention recent work on interfaces [8] and models such as ANNNI, Potts, etc... [5, 10, 4$]$.

What all the above approaches have in common, is the hypothesis of translation invariance (or periodicity) of the Hamiltonian. This assumption is used in many steps of the proofs and at a first glance it is not at all obvious whether it can be relaxed.

This paper is an extension of Pirogov-Sinai theory to the case of discrete and finite spin models on the lattice with finite range interactions, some of them being quasiperiodic; we require moreover the hamiltonian to have a finite number of periodic ground states. The main results without any proof were announced in a letter [11]; here we give all the details. The assumption is that the quasiperiodic part of the hamiltonian is a small perturbation of a periodic part. The extension we study here is not only of mathematical interest. Certain recently discovered alloys exhibit a quasiperiodic structure; it is interesting to understand the statistical properties of these materials. Models for thin epitaxial layers of two different species of adatoms on a quasiperiodic substratum fall into the class of interactions studied here. A related extension for statistical models on quasiperiodic lattices seems even more realistic; however, the statistical mechanics on such lattices is quite different and is actually under investigation. The case of periodic hamiltonians having no periodic ground states (see [16]) is treated by using Peierls argument in [13]; this case is not considered here. It would be interesting to extend the theory in the directions mentioned above.

One can think in terms of a concrete example where our extension applies. However, the assumptions that will be imposed on the interactions in order to prove the main theorem are valid on a much more general class of models. A concrete example is provided for instance by an Ising model on $\mathbb{Z}^{v}, v \geqq 2$ with 
hamiltonian

$$
H(\sigma)=\sum_{\langle s, t\rangle} \frac{1}{2}\left(1-\sigma_{s} \sigma_{t}\right)+\sum_{s} f_{s} \sigma_{s}
$$

where the field $f_{s}$ on each site $s \in \mathbb{Z}^{v}$ is a quasiperiodic variable, for instance of the type

$$
f_{t}=\alpha \sin \left(2 \pi \omega_{1} t_{1}+\cdots+2 \pi \omega_{v} t_{v}\right)
$$

with some small $\alpha$ and $\omega_{i} \in \mathbb{R}$, for $i=1, \ldots, v$.

The paper is organized as follows: In Sect. 2 we recall some definitions on quasiperiodic functions and give the definition of "gentleness." If the interaction potentials of the hamiltonian are smooth enough, and the frequencies $\omega_{i}$ are such that $\left\|n \omega_{i}\right\| \geqq 1 / K_{1}^{(i)} n^{2}$ for every non-zero integer $n$, the underlying small divisors problem can be treated in such a manner that a "gentleness" condition for the local ground energy densities $e_{q}(t)$ of the form

$$
\left|\sum_{i \in \Lambda} e_{q}(t)-\bar{e}\right| \Lambda|| \leqq K|\hat{c} \Lambda|
$$

can be proven. This fact is formalized by Lemma 1 of Sect. 2.

In Sect. 3 we recall the standard definitions of Pirogov-Sinai theory adapted to our case (without translation invariance).

Finally, in Sect. 4 we prove the technical steps that are needed to extend the Pirogov-Sinai theory to the case of quasiperiodic interactions. Lemma 1 of Sect. 2 is repeatedly used and we are able to prove the Main Theorem 5 as a corollary of the technical tools developed so far. This theorem states in a precise manner that the phase diagram at sufficiently low temperature is a homeomorphic deformation of the diagram at zero temperature.

The Main Theorem imposes a generalized Peierls condition on the contour weights. Assuming that the usual Peierls condition holds, only minor assumptions are needed to guarantee also the validity of the generalized Peierls condition. Rephrased in this way, the main theorem implies the following

Main Corollary. Consider a hamiltonian $H=H^{0}+H^{1}$ given by two classes of interactions: i) the "unperturbed," translation invariant (or periodic) part $H^{0}=\sum_{A: \mathrm{daam} \leqq r} \Phi_{A}^{0}\left(x_{A}\right)$, and ii) the "perturbation" $H^{1}=\sum_{\text {Adam } \leqq r} \Phi_{A}^{1}\left(x_{A}\right)$, where $\Phi_{A}^{1}$ are quasiperiodic potentials, all of them having the same frequency module generated by a generator $\omega$, and depending linearly on some vector parameter $\xi=\left(\xi_{1}, \ldots, \xi_{m}\right)$.

Let $\left\{x^{q}\right\}, q \in Q$ be the family of all ground states of $H^{0}$ which are constant (or periodic) configurations. Under the hypotheses:

H1. The Peierls condition

$$
H^{0}(x)-H^{0}\left(x^{q}\right) \geqq C|B(x)|,
$$

with $C>0$, holds, $B(x)$ being the set of all "incorrect points" $t \in \mathbb{Z}^{v}$ of $x$ (i.e. all points $t \in \mathbb{Z}^{v}$ such that there is no $x^{q}$ satisfying the condition $x=x^{q}$ on the $r$-neighborhood of $t$ ); H2. The quasiperiodic interactions, $\Phi_{A}^{1}$, are small for $\xi \in \mathscr{U} \subset \mathbb{R}^{m}$ and sufficiently smooth with respect to the quasiperiodicity, namely $\left|\partial^{3} \Phi_{A}^{1} / \partial(\omega t)^{3}\right| \leqq \varepsilon$ with a small $\varepsilon$, where $t$ is the lexicographically first element of $A$; 
H3. The frequency module generator $\omega$, satisfies the diophantine condition $\left\|n \omega_{i}\right\| \geqq$ $1 / K n^{2}$, for some coordinate $i=1, \ldots, v$;

H4. The temperature $T$ is sufficiently small; we prove that:

There is a homeomorphism $I: \mathscr{U} \rightarrow \tilde{\mathscr{U}}$, with $\mathscr{U} \subset \mathbb{R}^{m}$ and $\tilde{\mathscr{U}} \subset \mathbb{R}^{m}$, that maps the stratum of the zero temperature phase diagram

$$
E_{Q^{\prime}}=\left\{\xi \in \mathscr{U}: e_{q^{\prime}}(\xi)=\min _{q} e_{q}(\xi) \text { iff } q^{\prime} \in Q^{\prime}\right\}
$$

into the stratum

$H_{Q^{\prime}}=\left\{\xi \in \tilde{\mathscr{U}}: q^{\prime}\right.$ is stable for the hamiltonian $H(\xi)$ at temperature $T$ iff $\left.q^{\prime} \in Q^{\prime}\right\}$, for every subset $Q^{\prime} \subset Q$. By $e_{q}$ we denote the mean energy of $x^{q}$ and by "stability" of $q$ we mean that there is a q-like phase whose configurations attain the value $x^{q}$ in most points of $\mathbb{Z}^{v}$.

\section{Quasiperiodicity}

In this section we summarize some definitions on quasiperiodic functions [2]. Let us first introduce the notion of an almost periodic function.

Definition 1. A continuous function $f: \mathbb{R} \rightarrow \mathbb{R}$ is called almost periodic if for each $\varepsilon>0$ there exists a positive number $p=p(\varepsilon)$ such that any interval of the form $(t, t+p)$ contains at least one $s$ such that

$$
|f(r+s)-f(r)| \leqq \varepsilon, \text { for }-\infty<r<\infty .
$$

According to Bohr's fundamental theorem, any almost periodic function $f(r)$ on the additive group of real numbers can be expressed as uniform limit of linear combinations of the form $\sum C_{j} \exp \left(2 i \pi \lambda_{j} r\right)$ with $\lambda_{j} \in \mathbb{R}$.

The set $L_{f}$ generated by linear combinations of $\lambda_{j}$ 's with integer coefficients is called the frequency module of $f$.

Definition 2. A function $f: \mathbb{R} \rightarrow \mathbb{R}$ is quasiperiodic if it is almost periodic and its frequency module is finitely generated.

Example. Let $g: \mathbb{R}^{n} \rightarrow \mathbb{R}$ be a periodic function of period 1 in each variable. Let $\omega_{1}, \ldots, \omega_{n}$ be $n$ irrational numbers. Then $f(r)=g\left(\omega_{1} r, \ldots, \omega_{n} r\right)$ is a quasiperiodic function with frequency module $L_{f}=\left\{m_{1} \omega_{1}+\cdots+m_{n} \omega_{n} \mid m_{i} \in \mathbb{Z}\right\}$.

The above definitions can be extended to quasiperiodic functions $f: \mathbb{R}^{v} \rightarrow \mathbb{R}$. Introduce the vector notation for $m=\left(m_{1}, \ldots, m_{v}\right)$ and similarly for $\omega, t$, the scalar product $(t, \omega)=\sum_{i} \omega_{i} t_{i}$, and the componentwise product $m \omega=\left\{m_{1} \omega_{1}, \ldots, m_{v} \omega_{v}\right\}$. A function $f: \mathbb{R}^{v} \rightarrow \mathbb{R}$ is called quasiperiodic if it is quasiperiodic in each coordinate of its argument $t \in \mathbb{R}^{v}$. Similar definitions hold for almost periodic (respectively quasiperiodic) functions on $\mathbb{Z}^{v}[17]$.

We need now to explain where the quasiperiodicity enters into the problem. We state the relevant notions abstractly and give examples in terms of statistical physics. 
A field $\varphi$ is a function $\varphi: \mathbb{Z}^{v} \rightarrow A \subset \mathbb{R}$ (e.g. a spin configuration $\sigma$ is a field, assigning to every site $s \in \mathbb{Z}^{v}$ a value $\left.\sigma_{t} \in\{-1,1\}\right)$. For any set $W \subset \mathbb{Z}^{v}$, let $\left(t_{1}, \ldots, t_{|W|}\right)$ be the elements of $W$ taken in the lexicographic order. A functional $f_{W}$ of the field $\phi$ is called $W$-local if it depends only on the values the field $\varphi$ takes on $W$, i.e. it can be written as $f_{W}[\phi]=f\left(\varphi\left(t_{1}\right), \ldots, \varphi\left(t_{|W|}\right)\right)$. For example, the interaction potentials $\Phi_{A}$ are $A$-local functionals of the configurations.

Denote by $\operatorname{Fin}_{0}\left(\mathbb{Z}^{v}\right)$ the family of finite connected subsets of $\mathbb{Z}^{v}$ having as their lexicographically first element the point 0 . Fix some $W \in \operatorname{Fin}_{0}\left(\mathbb{Z}^{v}\right)$. For every $s \in \mathbb{Z}^{v}$, denote by $W_{s}$ the parallel translation of $W$ by $s$. Consider a $W_{s}$-local functional $f_{W_{s}}=f\left(\varphi\left(t_{1}-s\right), \ldots, \varphi\left(t_{\mid W_{s}}-s\right)\right)$. Let $g: F_{i n}\left(\mathbb{Z}^{v}\right) \times \mathbb{R}^{v} \times \mathbb{R}^{v} \rightarrow \mathbb{R}$ be a function that is periodic in the last vector variable with period $P=\left(P_{1}, \ldots, P_{v}\right) \in \mathbb{R}^{v}$. In the sequel we consider functions with period $P=(1, \ldots, 1)$ only. We say that a $W$-local functional $f_{W}$ is quasiperiodic with frequency module generated by a unique vector generator $\omega$, iff $f_{W_{s}}$ can be written as $f_{W_{s}}=g(W, \omega, \omega)$. We call $g$ the modulating function. As an example, consider the model defined in the Introduction. There, the one-particle interaction potential $\Phi_{i t\}}(\sigma)$ is given by $\Phi_{\{t\}}\left(\sigma_{t}\right)=f_{t} \sigma_{t}$, with $f_{t}=\alpha \sin 2 \pi(\omega, t)$; it is obviously a $\{l\}$-local quasiperiodic function. Generalizations to more complicated frequency modules are evident.

Remark. In order not to burden excessively the notation, it is understood in the whole paper that all functions of geometrical objects have this dependence on three variables. For instance if we write $F(\Gamma)$ in the following, it will be thought as a function of three variables. If $t$ is the lexicographically first point of $\operatorname{supp} \Gamma, F(\Gamma)$ means actually $F(\Gamma, \omega, \omega t)$. By the derivatives of $F$ with respect to $\omega t$ we mean the $n$-tuple of partial derivatives with respect to $\omega_{t} t_{i}$.

We prove the following:

Lemma 1. Assume that the modulating function $g(\cdot, \cdot, \cdot):$ Fin $_{0}\left(\mathbb{Z}^{v}\right) \times \mathbb{R}^{v} \times \mathbb{R}^{v} \rightarrow \mathbb{R}$ is $C^{3}$ with respect to the third variable. Assume that for a given $W \in \mathrm{Fin}_{0}\left(\mathbb{Z}^{v}\right)$ the third derivative with respect to the third variable has its $L^{\infty}$ norm bounded by some constant $K_{0}=K_{0}(W)$ for $W \in$ Fin $_{0}\left(\mathbb{Z}^{v}\right)$. Moreover, suppose that there is a coordinate $j \in\{1, \ldots, v\}$ of $\omega$ such that the following diophantine condition

$$
\left\|n \omega_{j}\right\| \geqq 1 / K_{1} n^{2}, \quad \forall n \in \mathbb{Z} \backslash\{0\}
$$

(where $\|t\|=\operatorname{dist}(t, \mathbb{Z})$ ) is fulfilled ${ }^{1}$. Then, there are two constants $K_{2}=K_{2}(W, \omega) \in \mathbb{R}$ and $K_{3}=K_{3}\left(W, \omega, K_{0}, K_{1}\right) \in \mathbb{R}^{+}$such that

$$
\left|\sum_{t \in \Lambda} g(W, \omega, \omega t)-K_{2}\right| \Lambda|| \leqq K_{3}|\partial \Lambda| .
$$

Proof. Without loss of generality one can assume that $j=v$ in the diophantine condition. For every vector $t \in \mathbb{Z}^{v}$, use the notation $\hat{t}$ to denote its $v-1$ first

\footnotetext{
1 This condition implies that there is a constant $K_{1}^{\prime}$ slightly larger than $K_{1} / \pi$ such that $\left|\sin \left(n \omega_{j} \pi\right)\right|$ $\geqq 1 / K_{1}^{\prime} n^{2}$ for every non-zero integer
} 
coordinates; hence, $t=\left(\hat{t}, t_{v}\right)$. Take the Fourier transform of $g$

$$
\begin{aligned}
g(W, \omega, \omega t) & =\sum_{n \in Z} \tilde{g}_{n}(W, \omega, \widehat{\omega t}) \exp \left(i \pi n \omega_{v} t_{v}\right) \\
& =\tilde{g}_{0}(W, \omega, \widehat{\omega t})+\sum_{n \neq 0} \tilde{g}_{n}(W, \omega, \widehat{\omega t}) \exp \left(i \pi n \omega_{v} t_{v}\right) .
\end{aligned}
$$

Then, using the diophantine condition above we estimate

$$
\left|\sum_{t_{v} \in[a, b]} \exp \left(i \pi n \omega_{v} t_{v}\right)\right| \leqq 2\left|\sin \left(\pi n \omega_{v}\right)\right|^{-1} \leqq 2 K_{1}^{\prime} n^{2}
$$

and

$$
\begin{aligned}
\left|\sum_{t \in \Lambda}\left[g(W, \omega, \omega t)-\tilde{g}_{0}(W, \omega, \omega t)\right]\right| & \leqq \sum_{\hat{i}: \exists t \in \Lambda}\left|\sum_{n \neq 0} \tilde{g}_{n}(W, \omega, \hat{\omega t}) \sum_{t_{v}:\left(\hat{t}, t_{v}\right) \in \Lambda} \exp \left(i \pi n \omega_{v} t_{v}\right)\right| \\
& \leqq \sum_{\hat{t} \cdot \exists t \in \Lambda} 2 K_{1}^{\prime} \sum_{n \neq 0}\left|\tilde{g}_{n}\right| N_{\hat{t}} n^{2},
\end{aligned}
$$

where $N_{\hat{t}}$ is the number of the vectors $\hat{t}$, such that $\left(\hat{t}, t_{v}\right) \in \partial \Lambda$ for some $t_{v}$.

As $\sum_{\hat{t}: \exists t \in \Lambda} N_{\hat{t}} \leqq|\partial \Lambda|$ we can further estimate

$$
\begin{aligned}
\left|\sum_{t \in \Lambda}\left[g(W, \omega, \omega t)-\tilde{g}_{0}(W, \omega, \widehat{\omega t})\right]\right| & \leqq 2|\partial \Lambda| K_{1}^{\prime} \max _{\hat{t}} \sum_{n \neq 0}\left|\tilde{g}_{n}\right| n^{2} \\
& \leqq 2|\partial \Lambda| K_{1}^{\prime}\left(K_{0}\right)^{2}\left(\sum_{n \neq 0} \frac{1}{n^{2}}\right)^{1 / 2} .
\end{aligned}
$$

The last inequality is a consequence of the following observations:

$$
\sum_{n \neq 0}\left|\tilde{g}_{n} n^{2}\right| \leqq\left(\sum_{n \neq 0}\left|\tilde{g}_{n}^{2}\right| n^{6}\right)^{1 / 2}\left(\sum_{n \neq 0} \frac{1}{n^{2}}\right)^{1 / 2}
$$

by Hölder inequality and

$$
\sum_{n \neq 0}\left|\tilde{g}_{n}^{2}\right| n^{6} \leqq\left\|\partial^{3} g / \partial\left(\omega_{v} t_{v}\right)^{3}\right\|_{L^{2}}^{2}
$$

Now, since the period $P=\left(P_{1}, \ldots, P_{v}\right)=(1, \ldots, 1)$ we can bound $\left\|\partial^{3} g / \partial\left(\omega_{v} t_{v}\right)^{3}\right\|_{L^{2}}^{2} \leqq$ $\left\|\partial^{3} g / \partial\left(\omega_{v} t_{v}\right)^{3}\right\|_{L^{x}}^{2} \equiv K_{0}^{2}$.

Remark. The irrational numbers $\theta$ satisfying the typical condition $\|n \theta\| \geqq 1 / K_{1} n^{l}$ are called Diophantine numbers of type $l$ (in contradistinction to Liouville's numbers that are exponentially well approximated by rationals). The Diophantine numbers are not exceptional, in fact the Lebesgue measure of the reals that are not of Diophante's type is zero.

Definition 3. A function $G: \operatorname{Fin}_{0}\left(\mathbb{Z}^{v}\right) \times \mathbb{Z}^{v} \rightarrow \mathbb{R}$ of the form

$$
G(W, s)=g(W, \omega),(\omega s)
$$

such that for $g$ the hypotheses of the previous lemma are fulfilled and $\omega$ ) satisfies $\left\|n \omega_{j}\right\| \geqq 1 / K_{1} n^{2}$ for suitable $j$ and every $n \neq 0$, is called gentle, more precisely $\left(K_{0}, K_{1}\right)$-gentle, with $K_{0}=K_{0}(W)$ for $W \in$ Fin $_{0}\left(\mathbb{Z}^{v}\right)$. 


\section{Notations and Definitions}

Let us consider a discrete spin system on the lattice $\mathbb{Z}^{v}(v \geqq 2)$. A configuration $x$ is an element of the product space $S^{Z^{v}}$. For every finite subset $\Lambda \subset \mathbb{Z}^{v}$, we denote by $x_{\Lambda}$ the restriction of $x$ on $\Lambda$.

The theory is defined by a family of interaction potentials $\left\{\Phi_{A}\right\}_{A \subset Z^{v}}$, i.e. local functions of the type $\Phi_{A}: S^{A} \rightarrow \mathbb{B} \cup\{+\infty\}$. We consider only finite range potentials. We do not ask all the potentials to be translationally invariant; some of them are allowed to vary quasiperiodically as we explain later. Given a finite volume $\Lambda$ and a fixed boundary condition $x_{\Lambda^{c}}$ on $\Lambda^{c}$, the relative hamiltonian $H\left(x_{\Lambda} \mid x_{\Lambda^{c}}\right)$ for every configuration $x_{A}$ is defined by

$$
H\left(x_{\Lambda} \mid x_{\Lambda^{c}}\right)=\sum_{A \notin \Lambda^{c}} \Phi_{A}\left(x_{A}\right)
$$

and the corresponding partition function by

$$
Z\left(\Lambda, x_{\Lambda^{c}}\right)=\sum_{x_{\Lambda} \in S^{\Lambda}} \exp \left(-H\left(x_{\Lambda} \mid x_{\Lambda^{c}}\right)\right) .
$$

The inverse temperature $\beta$ is included as a multiplicative factor in $\Phi_{A}$.

The hamiltonian will be now expressed in terms of contours. For a general introduction to the contour machinery the reader may consult [18]. Here, some additional definitions are given in order to include the case of non-translation invariance.

The notion of reference configurations is defined in such a way that the finite temperature states are obtained as small perturbations of some of these reference configurations. There is no a priori restriction in their choice. However, the set of reference states must include all the local ground states to insure that the Peierls condition is fulfilled. The set of constant reference configurations obtained as the ground states of the unperturbed periodic part of the hamiltonian, provides a typical example.

Let $\left\{x^{q}, q \in Q\right\}$ be the set of reference states with $Q$ some index set. We require $|Q|<\infty$.

Let some distance be defined on $\mathbb{Z}^{v}$. Denote by $B_{t}(r)$ the sphere of radius $r$ and center $t$ with respect to that distance, $r$ being the range of interactions. A point $t \in \mathbb{Z}^{v}$ such that $x_{t}=x_{s}^{q}, \forall s \in B_{t}(r)$ is called a $q$-correct point for the configuration $x$. Consequently, a point $t \in \mathbb{Z}^{v}$ is an incorrect point for $x$ if there is no $q \in Q$ for which $t$ is $q$-correct. The set of all incorrect sites of $x$ is denoted by $B(x)$.

Definition 4. Let $C$ be a finite connected component of $B(x)$. A contour $\Gamma$ for the configuration $x$, is the pair $\Gamma=\left(x_{C}, C\right)$, where $x_{C}$ is the restriction of the configuration $x$ to the component $C$. $C$ is usually denoted by supp $\Gamma$.

The hamiltonian (1) will now be reformulated in terms of contours. The contour having $q$-correct points as its exterior is denoted by $\Gamma^{q}$. Each finite component of $(\operatorname{supp} \Gamma)^{c}$ is characterized by some index $q^{\prime} \in Q$. The union of all components with the same $q^{\prime}$ is denoted by $\operatorname{int}_{q^{\prime}} \Gamma$. We denote by int $\Gamma=\bigcup_{q^{\prime} \in Q} \operatorname{int}_{q^{\prime}} \Gamma$ and $V(\Gamma)=$ $\operatorname{supp} \Gamma \cup \operatorname{int} \Gamma$, ext $\Gamma=V(\Gamma)^{c}$. 
For each reference state, the local energy densty $e_{q}(t)$ is defined by

$$
e_{q}(t)=\sum_{A \ni t} \frac{\Phi_{A}\left(x^{q}\right)}{|A|}, \text { for } t \in \mathbb{Z}^{v},
$$

and the contour weight is defined by

$$
\Phi\left(\Gamma^{q}\right)=\sum_{A \subset Z^{v}} \Phi_{A}\left(x_{A}\right) \frac{\left|A \cap \operatorname{supp} \Gamma^{q}\right|}{|A|}-\sum_{t \in \text { supp } \Gamma^{q}} e_{q}(t) .
$$

Fix a boundary condition $x_{A^{c}}^{q}$. Then, assuming that all contours $\Gamma_{i}$ of $x_{A} \cup x_{A^{c}}^{q}$ satisfy the condition supp $\Gamma_{i} \subset \Lambda$, we have for the hamiltonian the expression [20]

where

$$
H\left(x_{\Lambda} \mid x_{\Lambda^{\prime}}^{q}\right)=\sum_{i} \Phi\left(\Gamma_{i}\right)+\sum_{q^{\prime} \in Q} \sum_{t \in \Lambda_{q^{\prime}}} e_{q^{\prime}}(t)+\operatorname{const}(\Lambda, q),
$$

$$
\hat{\Lambda}_{q}=\Lambda_{q} \bigcup_{i} \operatorname{supp} \Gamma_{i}^{q},
$$

and $\Lambda_{q}$ is the set of $q$-correct points contained in $\Lambda$. We will replace $H\left(x_{A} \mid x_{\Lambda^{c}}\right)$ by the quantity $H\left(x_{A}\right)=\sum_{i} \Phi\left(\Gamma_{i}\right)+\sum_{q^{\prime} \in Q} \sum_{t \in \Lambda_{q^{\prime}}} e_{q^{\prime}}(t)$ everywhere in the following. As $H\left(x_{\Lambda} \mid x_{\Lambda^{c}}^{q}\right)-H\left(x_{\Lambda}\right)=$ const $(\Lambda, q)$ this substitution does not affect the values of the Gibbs probabilities we will work with.

The family $\left\{\Gamma_{i}\right\}$ of contours associated with a given configuration has an intrinsic partial order induced by the inclusion relation of the supports, in the following sense: A contour $\Gamma_{1}$ is smaller than $\Gamma_{2}$ if dist $\left.\left(\operatorname{supp} \Gamma_{1} \text {, (int } \Gamma_{2}\right)^{c}\right) \geqq 2$. The maximal elements in this ordering are called external contours of the given configuration.

Definition 5. A contour weight $\Phi(\cdot)$ satisfies the Peierls-Gertzik-Pirogov-Sinai (P-GPS) condition if there is some large $\tau>0$ such that

$$
\Phi(\Gamma)>\tau|\operatorname{supp} \Gamma| \text { for every } \Gamma \text {. }
$$

Remark. As the inverse temperature $\beta$ is included in $\Phi$, the parameter $\tau$ is a function of $\beta$, and grows linearly with $\beta$.

A configuration $x_{\Lambda}$ is called $q$-diluted in $\Lambda$ if its extension by $x^{q}$ on $\mathbb{Z}^{v}$ has the property that $\operatorname{dist}\left(V(\Gamma), \Lambda^{c}\right) \geqq 2$ for every contour $\Gamma$ of the extended configuration $x_{\Lambda} \cup x_{\Lambda^{c}}^{q}$. The corresponding partition function, i.e.

$$
Z^{q}(\Lambda, H)=\sum \exp \left(-H\left(x_{\Lambda}\right)\right)
$$

where the summation is over all the $q$-diluted configurations in $\Lambda$ is called $q$-diluted partition function in $\Lambda$.

We denote by

$$
Z\left(\Gamma^{q}, H\right)=\sum \exp \left(-H\left(x_{V(\Gamma)}\right)\right)
$$

the partition function where the summation is over all configurations on $V(\Gamma)$ which can be extended by $x^{q}$ on $\mathbb{Z}^{v}$ such that $\Gamma$ is their unique external contour.

The reference partition function $Z_{\mathrm{ref}}\left(\Gamma^{q}, H\right)$ is defined as the sum

$$
Z_{\text {ref }}\left(\Gamma^{q}, H\right)=\sum \exp \left(-H\left(x_{V(\Gamma)}\right)\right)
$$


over all configurations on $V(\Gamma)$ such that all the contours of the extended configuration $x_{V\left(\Gamma^{c}\right.}$ are disconnected from supp $\Gamma$.

In the particular case where int $\Gamma^{q}=$ int $_{q} \Gamma^{q}$, it happens that $Z\left(\Gamma^{q}, H\right)=$ $\exp \left(-\Phi\left(\Gamma^{q}\right)\right) Z_{\text {ref }}\left(\Gamma^{q}, H\right)$. Hence $\Phi(\Gamma)$ is interpreted as the work needed to install $\Gamma$. For a general $\Gamma$, such an interpretation can be given to the following quantity:

Definition 6. For every contour $\Gamma$ we define the physical contour functional $F_{\text {phys }}(\Gamma)$ as

$$
F_{\text {phys }}(\Gamma)=-\log \left(Z(\Gamma, H) / Z_{\text {ref }}(\Gamma, H)\right) .
$$

Now we can define the abstract contour model.

Assume that some contour functional $F$, acting on all contours $\Gamma^{q}$, is given, e.g. $F=F_{\text {phys }}$.

Definition 7. Let $\mathscr{K}^{q}{ }_{\Lambda}$ be the family of all $q$-contours that are diluted in $\Lambda$ in the sense that $\operatorname{dist}\left(\operatorname{supp} \Gamma, \Lambda^{c}\right) \geqq 2$, for $\forall \Gamma \in \mathscr{K}^{q}{ }_{\Lambda}$. Let $\mathscr{F}{ }_{\Lambda}^{q}$ be the family of all forests of contours in $\mathscr{K}^{q}{ }_{\Lambda}$ (we say that a set $W$ of contours is a forest if any two contours $\Gamma$, $\Gamma^{\prime}$ in $W$ have $\left.\operatorname{dist}\left(\operatorname{supp} \Gamma, \operatorname{supp} \Gamma^{\prime}\right) \geqq 2\right)$. Let $k: \mathscr{K}^{q}{ }_{\Lambda} \rightarrow \mathbb{R}$ be defined by $k(\Gamma)=$ $\exp (-F(\Gamma))$. We define by $Z_{\Lambda}^{q}(F)$ the partition function of the abstract polymer model by

$$
Z_{\Lambda}^{q}=\sum_{W \in \mathscr{F}_{A}^{q}} \prod_{\Gamma \in W} k(\Gamma)
$$

The cluster expansion technique can be applied to the study of these contour models, if $F$ is a $\rho$-functional (i.e. $F(\Gamma) \geqq \rho|\operatorname{supp} \Gamma|$ holds for every $q$-contour $\Gamma$ ) with a sufficiently large $\rho$. Then, by using standard cluster expansion arguments [12] the cluster expansion series for $\log Z_{\Lambda}^{q}$ converges if $\rho$ is sufficiently large. However, for a physical contour functional the cluster expansion does not converge in general. To handle these difficulties we will introduce some metastable contour model in Sect. 4 replacing the physical model.

\section{Extension of the Pirogov-Sinai Theory}

\subsection{Basic Constructions and Estimates}

We start by presenting without proof a theorem establishing the equivalence between the physical model and the polymer model. This equivalence is established only in the case of the physical contour functional. The proof can be easily done by induction on the level of $\Lambda$ (see [20]; some obvious changes are needed to get rid of the lack of translation invariance). In the following, by a contour functional $F$ we mean a family of functionals $F_{q}$, each $F_{q}$ acting on all $q$-contours $\Gamma^{q}$.

Theorem 1. If $F_{\text {phys }}$ is the physical contour functional given by (2), then for each $q$ and each $\Lambda$ with simply connected components we have

$$
Z^{q}(\Lambda, H)=\exp \left(-\sum_{t \in \Lambda} e_{q}(t)\right) Z_{\Lambda}^{q}\left(F_{\text {phys }}\right) .
$$

Up to this point, the exposition was very similar to the standard Pirogov-Sinai 
theory. The lack of translation invariance introduced only minor apparent changes (namely $e_{q}(t)$ is a function of the point $t$ ) and some hidden changes (namely all the functions of contours $\Phi, F$ etc. depend not only on the shape of the contour and the configuration on it but also on its position). The following technical steps allow us to cope with the lack of translation invariance.

Lemma 2. Let $F_{q}$ be a $\rho$-contour functional. Let moreover $\exp \left(-F_{q}\left(\Gamma^{q}\right)\right)$ be a gentle function (see definition 3) whose derivatives up to the third order with respect to the third variable are bounded by $\exp (-\rho|\operatorname{supp} \Gamma|)$ with $\rho$ sufficiently large. Then, for every $\Lambda$ we have

$$
\left|\log Z_{\Lambda}^{q}\left(F_{q}\right)-s_{q}\left(F_{q}\right)\right| \Lambda|| \leqq K_{4}|\partial \Lambda|
$$

and

$$
\left|s_{q}\left(F_{q}\right)\right| \leqq K_{4},
$$

where $-s_{q}\left(F_{q}\right)$ is the free energy of the polymer model defined by

$$
s_{q}\left(F_{q}\right)=\lim _{\Lambda \uparrow Z^{\prime}} \frac{\log Z_{\Lambda}^{q}\left(F_{q}\right)}{|\Lambda|}
$$

and $K_{4} \rightarrow 0$ if $\rho \rightarrow \infty$.

Proof. Expand

$$
Z_{\Lambda}^{q}(F)=\sum_{W \in \tilde{F}_{\Lambda}} \prod_{\Gamma \in W} \exp (-F(\Gamma))
$$

using standard cluster methods. Then $\log Z_{A}^{q}$ can be written as a sum over cluster products,

$$
\log Z_{\Lambda}^{q}(F)=\sum_{T \in c^{\Lambda}} k_{T}
$$

where $c_{c}$ means connected subset of $\Lambda$ which is disconnected from $\Lambda^{\mathrm{c}}$ and $k_{T}=\sum_{\left\{\Gamma_{1}\right\} \in \mathscr{F}(T)} \prod_{i} \exp \left(-F\left(\Gamma_{2}\right)\right) ; \mathscr{F}(T)$ is the collection of some chains (not to be specified here; see e.g. $[6,12]$ for details) of contours $\left\{\Gamma_{l}\right\}$ such that $\cup_{i} \operatorname{supp} \Gamma_{i}=T$ (we say a family $\left\{\Gamma_{i}\right\}$ of contours is a chain if $\operatorname{dist}\left(\operatorname{supp} \Gamma_{i}, \cup_{j<i} \operatorname{supp} \Gamma_{j}\right.$ ) $\leqq 1$, for all $i \geqq 2$ ). We require moreover the collection $\mathscr{T}(T)$ to be invariant with respect to the shifts i.e. $\mathscr{T}(T)_{s}=\mathscr{T}\left(T_{s}\right)$. (See e.g. [12] for details.) Now, the functions $\exp (-F(\Gamma))$ being $\left(\exp (-\rho|\operatorname{supp} \Gamma|), K_{1}\right)$-gentle, the cluster products $k_{T}$ will be $\left.(\exp (-(\rho-\zeta) T)), K_{1}\right)$-gentle functions, where $\zeta=\zeta(v)$. Put

$$
s_{q}(t, F)=\sum_{T \ni t}|T|^{-1} k_{T}
$$

and

$$
s_{q}(F)=\lim _{\Lambda \uparrow Z^{v}} \frac{1}{|\Lambda|} \log Z_{\Lambda}^{q}(F)=\lim _{\Lambda \uparrow Z^{v}} \frac{1}{|\Lambda|} \sum_{t \in \Lambda} s_{q}(t, F)
$$

(the mean value of $s_{q}(t, F)$ ). Now apply Lemma 1 ; because $|\operatorname{supp} \Gamma| \geqq 2 v+1$ for any contour $\Gamma$, both $s_{q}$ and $K_{4}$ are of the order $K_{1} \exp (-((2 v+1) \rho-\zeta))$, where $\zeta=\zeta(v)$. 
Unfortunately, it will not be possible, in general, to put $F_{q}=F_{\text {phys }}$ in the Lemma (2) above. Some more careful choices of $F_{q}$ will be needed. Before we define them, we need the following

Definition 8. Assume that the P-GPS condition (Definition 5) is fulfilled. Choose some $\tau_{1}<\tau$, to be specified later. A contour $\Gamma^{q}$ will be called stable if the following holds:

$$
\sum_{q_{1} \in Q}\left(\log Z^{q_{2}}\left(\operatorname{int}_{q_{1}} \Gamma, H\right)-\log Z^{q}\left(\operatorname{int}_{q_{1}} \Gamma, H\right)\right) \leqq \tau_{1}\left|\operatorname{supp} \Gamma^{q}\right|
$$

for any choice of $q_{2}=q_{2}\left(q_{1}\right)$. Moreover, $\Gamma^{q}$ will be called a small contour if it is hereditary stable (i.e. it is stable and all the $q$-contours contained in its interior are stable). The choice of $\tau_{1}$ will be specified below.

Remark. For a typical contour $\Gamma^{q}$ we have int $\Gamma^{q}=\operatorname{int}_{q_{1}} \Gamma^{q}$ for some $q_{1} \neq q$. The left-hand side of (5) is a measure of the volume gain for the free energy. By stability of $\Gamma$ we mean that this volume effect is "considerably smaller" than the energy of the contour.

There are several possible variants of the notion of stability; what is uniquely defined - and has a physical interpretation - is only the notion of a stable $q$ (see below).

Let us proceed in the discussion of those $F$ which will be used in the following. From now on, we shall consider a special class of contour functionals $F$ satisfying the following conditions:

i) $F(\Gamma)=F_{\text {phys }}(\Gamma)$ for any small contour $\Gamma$.

ii) $F$ is a $\rho$-functional with some large $\rho$.

iii) $F \geqq F_{\text {phys }}$ for any $\Gamma$.

Later, we shall explain in detail the construction of such an $F$ and show that $F^{q}=F_{\text {phys }}^{q}$ for stable $q$.

Define $h_{q}(t, F)=e_{q}(t)-s_{q}(t, F)$ and the mean quantities

$$
h_{q}(F)=\lim _{\Lambda \uparrow Z^{v}} \frac{1}{|\Lambda|} \sum_{t \in \Lambda} h_{q}(t, F) .
$$

Put $h(F)=\min _{q} h_{q}(F)$ and introduce

$$
a_{q}(F)=h_{q}(F)-h(F)
$$

that will be interpreted as "parameters of instability." Those $q$ for which $a_{q}=0$ will be called the stable q.

Definition 9. We say that a contour functional $F$ is $\left(\rho, K_{1}\right)$-gentle if each $\exp (-F(\Gamma))$ is a $\left(\exp (-\rho|\operatorname{supp} \Gamma|), K_{1}\right)$-gentle function of $\Gamma$.

The following theorem provides useful bounds on the partition functions.

Theorem 2. Let the function $e_{q}(\cdot)$ be $\left(K_{0}, K_{1}\right)$-gentle. Let $F$ be a $\left(\rho, K_{1}\right)$-gentle functional with sufficiently large $\rho$, such that $F(\Gamma) \geqq F_{\text {phys }}(\Gamma)$ for all contours $\Gamma$, and $F(\Gamma)=F_{\text {phys }}(\Gamma)$ for all small contours $\Gamma$. Then the free energy $h(F)$ does not depend on $F$. It equals the free energy $h$ of the given model. Moreover, the following assertions are true: 
i) unstable contours satisfy the inequality:

$$
a_{q}(F) \mid \text { int } \Gamma^{q}\left|\geqq\left(\tau_{1}-2 \varepsilon\right)\right| \operatorname{supp} \Gamma^{q} \mid,
$$

ii) $Z^{q}(\Lambda, H) \geqq \exp \left(-h_{q}(F)|\Lambda|-\varepsilon\left|\partial \Lambda^{c}\right|\right)$,

iii) $Z^{q}(\Lambda, H) \leqq \exp \left(-h(F)|\Lambda|+\varepsilon\left|\partial \Lambda^{c}\right|\right)$

with $\varepsilon=\varepsilon(\rho)$ and $\lim \varepsilon=0$.

$$
\underset{\substack{\rho \rightarrow \infty \\ K_{0} \rightarrow 0}}{ }
$$

Proof. First we prove ii). Write $Z^{q}(\Lambda, H)=\exp \left(-\sum_{t \in \Lambda} e_{q}(t)\right) Z_{\Lambda}^{q}(F)$. The function $e_{q}(\cdot)$ being $\left(K_{0}, K_{1}\right)$ - gentle, the use of Lemma 2 and Lemma 1 insures the validity of ii).

Next we prove i). If $\Gamma^{q}$ is unstable then by definition (5),

$$
\tau_{1}\left|\operatorname{supp} \Gamma^{q}\right| \leqq \sum_{q_{1}}\left(\log Z^{q_{2}}\left(\text { int }_{q_{1}} \Gamma, H\right)-\log Z^{q}\left(\text { int }_{q_{1}} \Gamma^{q}, H\right)\right)
$$

for some $q_{2}$. Using ii) and inductively iii) for smaller volumes we prove

$$
\left(h_{q}(F)-h(F)\right)\left|\operatorname{int} \Gamma^{q}\right|>\left(\tau_{1}-2 \varepsilon\right)|\operatorname{supp} \Gamma| .
$$

Now, we prove iii). For simplicity of the notations, we suppose that $|Q|=2$ (i.e. $\left.Q=\left\{q_{1}, q_{2}\right\}\right)$ and that only $q_{2}$ is stable. Then $a_{q_{1}}(F)>0$. Fix the family of external large contours $\left\{\Gamma_{i}\right\}$ and write int $=\bigcup_{i} \operatorname{int} \Gamma_{i}$, ext $=\Lambda \backslash \bigcup_{i} V\left(\Gamma_{i}\right)$. Write $Z^{q_{1}}\left(\Lambda,\left\{\Gamma_{i}\right\}\right)=\sum \exp (-H(x))$, where the sum is over all configurations in $\Lambda$ having $\left\{\Gamma_{i}\right\}$ as their unique family of external large contours. We can use the equivalence with the polymer model in the volume ext since only small contours can appear there. The summation over the diluted configurations in ext is bounded by $\exp \left(-h_{q_{1}}(F)|\operatorname{ext}|+\varepsilon\left|\partial \operatorname{ext}^{c}\right|\right)$ since it involves only small contours (use again the assumption that $F=F_{\text {phys }}$ for small $\Gamma$ ). The summation over the diluted configurations in int is bounded by $\exp \left(-h(F) \mid\right.$ int $|+\varepsilon| \partial$ int $\left.^{c} \mid\right)$ by using inductively the relation iii) in a smaller volume. Then

$$
\begin{aligned}
Z^{q_{1}}\left(\Lambda,\left\{\Gamma_{i}\right\}\right) \leqq & \exp \left(-h_{q_{1}}(F)|\operatorname{ext}|-h(F)|\operatorname{int}|-\sum_{i} \Phi\left(\Gamma_{i}\right)\right) \\
& \cdot \exp \left(2 \varepsilon \sum_{i}\left|\operatorname{supp} \Gamma_{i}\right|+\varepsilon\left|\partial \Lambda^{c}\right|\right) .
\end{aligned}
$$

Define $\Phi^{\prime}(\Gamma)=\Phi(\Gamma)-2 \varepsilon|\operatorname{supp} \Gamma|$, and suppose that $\Phi^{\prime}(\cdot)$ fulfills the P-G.P.S. condition with some new large $\tau_{2} \leqq \tau$. Now, $Z^{q_{1}}(\Lambda, H)$ can be estimated by the following sum over the families of external large contours:

$$
Z^{q_{1}}(\Lambda, H) \leqq \exp \left(-h(F)|\Lambda|+\varepsilon\left|\partial \Lambda^{c}\right|\right) \sum_{\left\{\Gamma_{l}\right\}} \exp \left(-a(F)|\operatorname{ext}|-\sum_{i} \Phi^{\prime}\left(\Gamma_{i}\right)\right) .
$$

Using the Lemma 3 below we can bound

$$
\sum_{\left\{\Gamma_{l}\right\}} \exp \left(-a(F)|\operatorname{ext}|-\sum_{i} \Phi^{\prime}\left(\Gamma_{i}\right)\right) \leqq \exp \left(\varepsilon\left|\partial \Lambda^{c}\right|\right)
$$

if $a(F)>s\left(\Phi^{\prime}\right)$. The quantity $-s\left(\Phi^{\prime}\right)$ - not to be confused with $-s(F)$ - is roughly 
speaking, the free energy of the polymer model with activities $\exp \left(-\Phi^{\prime}(\Gamma)\right)$ and only with large contours permitted. This condition on $a$ that is necessary for the validity of Lemma 3, can be easily verified in our case. The proof is based on the inductive assumption and the fact that $\exp \left(-\tau_{1} / a\right) \ll a$. Thus

$$
Z^{q_{1}}(\Lambda, H) \leqq \exp \left(-h(F)|\Lambda|+\varepsilon\left|\partial \Lambda^{c}\right|\right) .
$$

Let us state without proof [20] the lemma used at the last step of the previous theorem.

Lemma 3. Define an auxiliary model of external contours by assigning to every collection of compatible external contours $\left\{\Gamma_{i}\right\}$ in $\Lambda$ a "hamiltonian"

$$
H\left(\left\{\Gamma_{i}\right\}\right)=a|\operatorname{ext}|+\sum_{i} \Phi^{\prime}\left(\Gamma_{i}\right)
$$

with ext $=\Lambda \backslash \cup_{i} V\left(\Gamma_{i}\right)$ and $\Phi^{\prime}(\Gamma)>\tau|\operatorname{supp} \Gamma|$ with $\tau$ large. Then, if $s\left(\Phi^{\prime}\right)<$ a, we have

$$
\sum_{\left\{\Gamma_{i}\right\}} \exp \left(-H\left(\left\{\Gamma_{i}\right\}\right) \leqq \exp \left(\varepsilon_{1}\left|\partial \Lambda^{c}\right|\right)\right.
$$

\subsection{Construction of the Contour Functional}

The problem which is not yet solved is how to find some $F$ satisfying the conditions of Theorem 2. Once such an $F$ is found, we interpret $h_{q}(F)$ as the free energy of some metastable contour model, the relation $h_{q}=h$ meaning that $x^{q}$ gives birth to a stable phase. The questions asked above are answered by Theorem 3 of this subsection that gives an explicit inductive construction of a contour functional $F$. This functional turns out to be gentle and satisfies the assumptions of the Theorem 2; it has henceforth a reasonable physical meaning.

We remark that our functions of geometrical objects (e.g. $\exp (-\Phi(\Gamma)))$ although non-translation invariant have a translation invariant functional dependence on their space-depending arguments. We introduce the symbolic notation $d / d \lambda$ for derivatives which have the following meaning: the operator $d / d \lambda$ acts on functions of geometrical objects and means derivatives with respect to $\omega s$, where $s$ is the first point of the corresponding geometrical object.

The main result of this subsection is a generalization of the corresponding theorem of [20] to our quasiperiodic situation. It is stated in the form of the following

Theorem 3. Let the function $e_{q}$ be $\left(\varepsilon, K_{1}\right)$-gentle. Let the potentials $\Phi$ be $\left(\tau, K_{1}\right)$-gentle, i.e. the following generalized P-GPS condition

$$
\left|\frac{d^{k}}{d \lambda^{k}} \exp (-\Phi(\Gamma))\right| \leqq \exp (-\tau|\operatorname{supp} \Gamma|) .
$$

holds for $k=0,1,2,3$. If $\tau$ is sufficiently large (depending on $K_{0}, K_{1}$ ) then there exists a functional $F$, such that $F \geqq F_{\text {phys }}$ for all contours, and $F=F_{\text {phys }}$ for any small $\Gamma$. Moreover, $F$ can be chosen such that it is $\left(\rho, K_{1}\right)$-gentle, namely

$$
\left|\frac{d^{k}}{d \lambda^{k}} \exp (-F(\Gamma))\right| \leqq \exp (-\rho|\operatorname{supp} \Gamma|)
$$

and $\lim _{\tau \rightarrow \infty} \rho=+\infty$. 
Proof. We shall construct inductively $F$ for larger and larger contours. Define $\Phi_{\Lambda}(\Gamma)$ (respectively $F_{\Lambda}(\Gamma)$ ) as the finite volume regularisation of $\Phi$ (respectively $F$ ), i.e.

$$
\Phi_{\Lambda}(\Gamma)= \begin{cases}\Phi(\Gamma) & \text { if } V\left(\Gamma_{s}\right) \subset \Lambda \text { for some shift } \Gamma_{s} \text { of } \Gamma \\ +\infty & \text { otherwise }\end{cases}
$$

and similarly for $F_{\Lambda}$. Denote by $h_{\Lambda}=\min h_{q}\left(F_{\Lambda}\right)$ the free energy of the restricted model with regularized interactions $\Phi_{\Lambda}(\stackrel{q}{\Gamma})$. We assume inductively that $F_{\Lambda}$ is given in such a manner that the Theorem 2 can be applied to this restricted model.

Let int $\Gamma^{q} \Gamma^{q}$ denote the set $\cup_{q^{\prime} \neq q}$ int $_{q^{\prime}} \Gamma^{q}$ and define

$$
\exp \left(-F\left(\Gamma^{q}\right)\right)=\frac{\exp \left(-\Phi\left(\Gamma^{q}\right)\right) \prod_{q^{\prime} \neq q} Z^{q^{\prime}}\left(\operatorname{int}_{*} \Gamma^{q}, H\right)}{\mathscr{M}\left(Z_{\mathrm{ref}}^{*}\left(\Gamma^{q}\right), \exp \left(-h_{V(\Gamma)}\right) \mid \text { int }_{*} \Gamma^{q}\left|-\tau_{1}^{\prime}\right| \operatorname{supp} \Gamma^{q} \mid\right)},
$$

where $\tau_{1}^{\prime}>\tau_{1}$ will be specified later and $\mathscr{M}\left(a_{\Gamma}, b_{\Gamma}\right)$ is a smooth version of the maximum defined e.g. as a $C^{3}$ function which is symmetric in its arguments and such that $\mathscr{M}\left(a_{\Gamma}, b_{\Gamma}\right) \geqq \max \left(a_{\Gamma}, b_{\Gamma}\right)$ and $\mathscr{M}\left(a_{\Gamma}, b_{\Gamma}\right)=a_{\Gamma}$ if $a_{\Gamma}>\exp (C|\operatorname{supp} \Gamma|) b_{\Gamma}$. (Notice that $\tau_{1}^{\prime}$ must be sufficiently greater than $\tau_{1}$, approximately like $\tau_{1}^{\prime} \geqq \tau_{1}+2 \varepsilon$ to guarantee that $F=F_{\text {phys }}$ for all small contours. On the other hand $\mathscr{M} \geqq \max$ guarantees $F \geqq F_{\text {phys }}$.) We shall prove inductively

i) that $F$ defined by (9) is gentle in the sense that it satisfies (8) with $\rho \simeq \tau-\tau_{1}$ and

ii) that (8) i.e. the gentleness of any new $F\left(\Gamma^{q}\right)$ defined by the formula (9) at the level $\Lambda$ implies that the Theorem 2 is valid for the next greater volume.

We sketch the induction on the volume.

Step 1. Suppose that $F(\Gamma)$ is a gentle functional satisfying the condition (8) above, for all $\Gamma$ such that $V(\Gamma) \subset \Lambda$. Then, for $\rho$ sufficiently large we have

$$
\left|\frac{d^{k}}{d \lambda^{k}} Z^{q}(\Lambda, H)\right| \leqq \exp \left(-h_{\Lambda}|\Lambda|+\varepsilon_{2}|\partial \Lambda|\right)
$$

with small $\varepsilon_{2}=\varepsilon_{2}(\varepsilon)$. In fact, writing $Z^{q}(\Lambda, H)$ in terms of families of external contours $\left\{\Gamma_{i}\right\}$,

$$
\begin{aligned}
Z^{q}(\Lambda, H)= & \sum_{\left\{\Gamma_{t}\right\}} \exp \left(-\sum_{t \in \operatorname{ext}} e_{q}(t)-\sum_{i}\left(\sum_{t \in \operatorname{supp} \Gamma_{i}} e_{q}(t)+\Phi\left(\Gamma_{i}\right)\right)\right) \\
& \cdot \prod_{i} \prod_{q_{1} \in Q} Z^{q_{1}}\left(\operatorname{int}_{q_{1}} \Gamma_{i}^{q}, H\right) .
\end{aligned}
$$

Where ext $\equiv \Lambda \backslash \cup_{i} V\left(\Gamma_{i}\right)$ we use item iii) of Theorem 2 to bound $Z^{q}\left(\Lambda^{\prime}, H\right)$ by $Z^{q}\left(\Lambda^{\prime}, H\right) \mid \leqq \exp \left(-h_{\Lambda^{\prime}}\left|\Lambda^{\prime}\right|+\varepsilon\left|\partial \Lambda^{\prime c}\right|\right)$. Then, using inductively (10) for all $\Lambda^{\prime} \subset \Lambda$ we bound

$$
\begin{aligned}
\left|\frac{d Z^{q}(\Lambda, H)}{d \lambda}\right| \leqq & \varepsilon|\Lambda| Z^{q}(\Lambda, H)+\sum_{\Gamma}\left(\frac{d \exp (-\Phi(\Gamma))}{d \lambda}\right) \prod_{q_{1}} Z^{q_{1}}\left(\operatorname{int}_{q_{1}} \Gamma, H\right) Z^{q}(\operatorname{ext} \Gamma, H) \\
& +\sum_{\Gamma} \frac{d \prod_{q_{1}} Z^{q_{1}}\left(\operatorname{int}_{q_{1}} \Gamma, H\right)}{d \lambda} \exp (-\Phi(\Gamma)) Z^{q}(\operatorname{ext} \Gamma, H)
\end{aligned}
$$




$$
\begin{aligned}
& \leqq \varepsilon|\Lambda| \exp \left(-h_{\Lambda}|\Lambda|+\varepsilon|\partial \Lambda|\right) \\
& \quad+2 \sum_{\Gamma: \operatorname{dist}\left(\operatorname{supp} \Gamma \cdot \Lambda^{c}\right) \geqq 2} \exp (-(\tau-\varepsilon)|\operatorname{supp} \Gamma|) \exp \left(-h_{\Lambda}|\Lambda|\right) \\
& \leqq \\
& \exp \left(-h_{\Lambda}|\Lambda|+\varepsilon|\partial \Lambda|\right)
\end{aligned}
$$

The higher order derivatives generate a sum of monomials of the type appearing in (12); each monomial appearing in this case can be bounded as in (12) since it refers to smaller volumes.

Step 2. We show that $F(\Gamma)$ defined by (9) is bounded as in (8) if this is true for all $\Gamma^{\prime}$ that can fit in $V(\Gamma)$. Write

$$
\begin{aligned}
\frac{d}{d \lambda} \exp \left(-F\left(\Gamma^{q}\right)\right)= & \frac{d}{d \lambda} \exp \left(-\Phi\left(\Gamma^{q}\right) \prod_{q^{\prime} \neq q} Z^{q^{\prime}}\left(\operatorname{int}_{*} \Gamma^{q}, H\right)\right) \\
& \cdot\left[\mathscr{M}\left(Z_{\mathrm{ref}}^{*}\left(\Gamma^{q}\right), \exp \left(-h_{V(\Gamma)}\left|\operatorname{int}_{*} \Gamma^{q}\right|-\tau_{1}^{\prime}\left|\operatorname{supp} \Gamma^{q}\right|\right)\right]^{-1}\right. \\
& -\frac{\exp \left(-\Phi\left(\Gamma^{q}\right)\right) \prod_{q^{\prime} \neq q} Z^{q^{\prime}}\left(\operatorname{int}_{*} \Gamma^{q}, H\right)}{\left[\mathscr{M}\left(Z_{\mathrm{ref}}^{*}\left(\Gamma^{q}\right)\right), \exp \left(-h_{V(\Gamma)} \mid \text { int }_{*} \Gamma^{q}\left|-\tau_{1}^{\prime}\right| \operatorname{supp} \overline{\left.\left.\Gamma^{q} \mid\right)\right]^{2}}\right.\right.} \\
& \cdot \frac{d}{d \lambda} \mathscr{M}\left(Z_{\mathrm{ref}}^{*}\left(\Gamma^{q}\right)\right), \exp \left(-h_{V(\Gamma)}\left|\operatorname{int}_{*} \Gamma^{q}\right|-\tau_{1}^{\prime}\left|\operatorname{supp} \Gamma^{q}\right|\right) .
\end{aligned}
$$

Estimate this by using (7) and (10). We don't care about signs but we bound by the worst sum of positive terms. We bound $d \mathscr{M} / d \lambda$ by

$$
\frac{d \mathscr{M}}{d \lambda} \leqq C^{\prime}\left(\frac{d}{d \lambda} Z_{\mathrm{ref}}^{*}\left(\Gamma^{q}\right)+\frac{d}{d \lambda} \exp \left(-h_{V(\Gamma)}\left|\operatorname{int}_{*} \Gamma\right|-\tau_{1}^{\prime}\left|\operatorname{supp} \Gamma^{q}\right|\right)\right)
$$

Hence we need bounds on $d h_{V(\Gamma)} / d \lambda$ or equivalently on $d s\left(F_{V(\Gamma)}\right) / d \lambda$ in addition to the bound (10). But

$$
\frac{d s\left(F_{\Lambda}\right)}{d \lambda}=\lim _{\Lambda \uparrow Z^{v}} \frac{1}{|\Lambda|} \sum_{t \in \Lambda_{\Gamma_{t}^{q}}} \sum_{\frac{d \exp \left(-F\left(\Gamma_{t}^{q}\right)\right)}{d \lambda}} \frac{Z_{\Lambda \backslash \operatorname{supp} \Gamma_{t}^{q}}(F)}{Z_{\Lambda}(F)} .
$$

This derivative is bounded by $C \exp (-(2 v+1) \rho)$ (notice that $|\operatorname{supp} \Gamma| \geqq 2 v+1)$ by virtue of the inductive step of the proof of (8) for $k=1$. Higher derivatives generate the same kind of terms as in (13), some of them having higher and higher powers in the denominator. The highest power in the denominator is 4 , henceforth it is enough to choose $\tau_{1}$ sufficiently smaller than $\tau / 4$, for example $2 \tau / 9$. If we choose further $\tau_{1}^{\prime}>\tau_{1}$ e.g. $\tau_{1}^{\prime}=4 \tau / 17$ and $\rho \simeq \tau-k \tau_{1}^{\prime}, k$ being the power of the denominator considered, all these terms can be bounded as above. This proves the gentleness of $\exp (-F(\Gamma))$ up to the level defined by $V(\Gamma)$.

In order to obtain the phase diagram (in the next subsection) we need also some control on the derivatives with respect to the external fields $\xi_{i}, i=1, \ldots, m$. In the case where the external fields do not modify the frequency module we can easily generalize the previous theorem into the following

Theorem 4. Suppose that the perturbed hamiltonian $H_{\xi}$ has the same frequency module as the hamiltonian $H_{\xi=0}$. Let the functions $\partial^{k} \partial^{l} e_{q} / \partial \lambda^{k} \partial \xi_{i}^{l}, k=0, \ldots, 3, l=0,1$ be 
$\left(K_{0}, K_{1}\right)$-gentle and $\partial^{k} e_{q} / \partial \lambda^{k}$ be $\left(\varepsilon, K_{1}\right)$-gentle. Let the functions $\hat{o}^{l} \exp (-\Phi(\Gamma)) / \partial \xi_{i}^{l}$, $l=0,1$ satisfy the gentleness condition

$$
\left|\frac{\partial^{k}}{\partial \lambda^{k}} \frac{\partial^{l}}{\partial \xi_{l}^{l}} \exp (-\Phi(\Gamma))\right| \leqq \exp (-\tau|\operatorname{supp} \Gamma|) .
$$

If $\tau$ is sufficiently large, then $F$ defined by the relation (9) satisfies

$$
\left|\frac{\partial^{k}}{\partial \lambda^{k}} \frac{\partial^{l}}{\partial \xi_{i}^{l}} \exp (-F(\Gamma))\right| \leqq \exp \left(-\rho^{\prime}|\operatorname{supp} \Gamma|\right)
$$

with $\lim _{\tau \rightarrow \infty} \rho^{\prime}=+\infty$.

Proof. The proof is some modification of the proof of Theorem 3 if one remarks the similarities between $\xi$ and $\lambda$ derivative in the course of the induction. Theorem 2 guarantees the existence of a "good functional" $F$ under the assumption of the $\left(\varepsilon, K_{1}\right)$-gentleness of $e_{q}$. In particular the partition function satisfies the bound of item iii) of Theorem 2. Now, rewrite all the steps of Theorem 3 where the regularized quantity $h_{A}$ is replaced by the real free energy of the model $h$. When we compute derivatives of expression of the form $\exp (-h|\Lambda|)$, there appear terms that are bounded by $K_{0}|\Lambda|$ (instead of terms that appeared when deriving $\exp \left(-e_{q}|\Lambda|\right)$ that were bounded by $\varepsilon|\Lambda|$ in Theorem 3). But even these bigger terms are beaten when combined with expressions of the form $\exp (-\tau|\partial \Lambda|)$ if the quantity $K_{0}$ is not excessively large.

Corollary 1. Under the conditions of the previous Theorem 4 for the derivatives of $\exp (-\Phi(\Gamma))$ and the densities $e_{q}(t)$, the quantities $s_{q}(F)$ and $d s_{q}(F) / d \xi_{i}$ are bounded by small constants.

\subsection{The Phase Diagram}

Suppose that the original hamiltonian admits $m+1$ degenerate states. Then the mean energy densities $e_{q}=\lim _{\Lambda \uparrow Z^{v}}(1 /|\Lambda|) \sum_{t \in \Lambda} e_{q}(t)$ will be the same for $q_{1}, \ldots, q_{m+1}$. Hence, at zero temperature we have the coexistence of $m+1$ states. We include the inverse temperature into the hamiltonian. By "zero temperature" we mean the limit case $\tau \rightarrow \infty$. To construct the phase diagram at zero temperature it is enough to introduce a set of $m$ external fields removing the degeneracy of the ground states, i.e. to introduce a vector parameter $\xi=\left(\xi_{1}, \ldots, \xi_{m}\right)$. However, we can only deal with those external perturbations that leave the frequency module of $H_{\xi}$ invariant for $\forall \xi \in \mathbb{R}^{m}$.

The mean energy densities become functions of $\xi, e_{q}=e_{q}(\xi)$ and the degeneracy $e_{q_{1}}(0)=\cdots=e_{q_{m+1}}(0)$ is removed in a sense explained in Theorem 5 below. The phase diagram can be described by the quantities $h_{q}(\xi)$. We consider the low temperature-large $\tau$-case; then these quantities are well defined from the previous considerations. In order to guarantee that we can continuously pass from the zero temperature diagram to the corresponding one at finite (low) temperature, the following two mappings

$$
z: \mathscr{U} \subset \mathbb{R}^{m} \rightarrow D^{m}, \quad z(\xi)=\left(e_{q_{1}}-e, \ldots, e_{q_{m+1}}-e\right), \text { with } \quad e=\min _{q} e_{q}
$$


and

$$
l: \tilde{\mathscr{U}} \subset \mathbb{R}^{m} \rightarrow D^{m}, \quad l(\xi)=\left(h_{q_{1}}-h, \ldots, h_{q_{m+1}}-h\right), \quad \text { with } \quad h=\min _{q} h_{q},
$$

where $D^{m}=\left\{\left(y_{1}, \ldots, y_{m+1}\right) \in \mathbb{R}^{m+1} ; \min y_{1}=0\right\}$ must be such that the mapping $l^{-1} \circ z: \mathscr{U} \rightarrow \tilde{\mathscr{U}}$ exists (for suitable $\mathscr{U}$ and $\tilde{\mathscr{U}}$ ) and is a homeomorphism near the identity. The homeomorphism $l^{-1} \circ z$ transforms the zero temperature diagram into the finite temperature one. Namely, if for some $\xi, N \subset Q$ is precisely the set of $q$ such that $e_{q}(\xi)=e(\xi)$, then for $\tilde{\xi}=l^{-1} \circ z(\xi), N$ is also the set of $q$ such that $h_{q}(\tilde{\xi})=h(\tilde{\xi})$. We can prove the following

Theorem 5. Let $|Q|=m+1$ and $\xi$ be a vector parameter written in the form $\left(\xi_{1}, \ldots, \xi_{m}\right)$. Let $e_{q}(\xi)$ be all the same for $\xi=0$ and the matrix

$$
M=\left(\frac{\partial\left(e_{q_{1}}-e_{q_{1}}\right)}{\partial \xi_{j}}\right),
$$

with $i=2, \ldots, m+1$ and $j=1, \ldots, m$ be invertible. Assume moreover that $\left\|M^{-1}\right\| \leqq L$ in some neighborhood $\mathscr{U}$ of 0 . Let the frequency module generator $\omega$ be an irrational number satisfying the diophantine property $\left\|n \omega_{j}\right\| \geqq 1 / K_{1} n^{2}$ for every non-zero integer $n$ and some coordinate $j \in\{1, \ldots, v\}$. Let the derivatives of the contour weight $\exp (-\Phi(\Gamma))$ be bounded

$$
\left|\frac{\partial^{k}}{\partial \lambda^{k}} \frac{\partial^{l}}{\partial \xi_{i}^{l}} \exp (-\Phi(\Gamma))\right| \leqq \exp (-\tau|\operatorname{supp} \Gamma|),
$$

and the local energy densities $e_{q}(t)$ satisfy the bound

$$
\left|\frac{\partial^{k}}{\partial \lambda^{k}} e_{q}(t)\right| \leqq \varepsilon
$$

with $\exp (\tau / 5) K_{0}^{-1} \gg 1$, for $k \in\{0,1,2,3\}$ and $l \in\{0,1\}$ and also the mild bounds

$$
\left|\frac{\partial^{k}}{\partial \lambda^{k}} \frac{\partial^{l}}{\partial \xi_{i}^{l}} e_{q}(t)\right| \leqq K_{0} \quad \text { for } \quad i=1, \ldots, m .
$$

If moreover $\tau=\tau(\mathscr{U}, L)$ is sufficiently large and $\varepsilon=\varepsilon(\mathscr{U}, L)$ sufficiently small, then the mapping

$$
l(\xi)=\left(h_{q_{1}}-h, \ldots, h_{q_{m+1}}-h\right)
$$

is invertible and one-to-one between some slightly smaller set $\tilde{\mathscr{U}} \subset \mathscr{U}$ and an open $\mathscr{V} \ni 0$ in $D^{m}$. The map $l^{-1} \circ z$, where $z$ is given by $z(\xi)=\left(e_{q_{1}}-e, \ldots, e_{q_{m+1}}-e\right)$ is moreover smooth, and transforms the zero temperature phase diagram (given by $\Phi(\Gamma)=\infty$ ) into the low temperature one.

The proof is an immediate consequence of the corollary (1) and the inverse mapping theorem.

The interpretation of the previous theorem is the following: The relation $h_{q}=h$ (and only this) means that there is a stable $q$-like phase $P_{q}$ of the given hamiltonian, having the following structure: $P_{q}$-almost any configuration $x$ satisfies the properties

i) all components of $B(x)$ are finite 
ii) external contours do exist; any $t \in \mathbb{Z}^{v}$ is either an external point to all contours of $x$ or $t \in V(\Gamma)$ for some external contour $\Gamma$ of $x$.

iii) $x=x^{q}$ on ext $=\mathbb{Z}^{v} \backslash \bigcup_{\text {all contours } \Gamma \text { of } x} V(\Gamma)$.

Intuitively, almost any configuration can be viewed as "a sea $x^{q}$ " with small, rare, isolated islands (the external contours of $x^{q}$ ) scattered uniformly over the whole $\mathbb{Z}^{v}$. The statistical properties of the ensemble of external contours are described exactly as in the corresponding $q$-contour model with activity $\exp \left(-F_{\text {phys }}\right)$ and $F_{\text {phys }}$ is a $\rho$-functional with a large $\rho$ in this case.

As a concluding remark let us mention that apart from the possible extensions that are under investigation, it is easy to generalize our results to take into account larger sets of irrational $\omega$ 's. However, the less restricting the conditions are on $\omega$, the more restricting the requirements should be on the derivability, i.e. if $\left\|n \omega_{i}\right\| \geqq 1 / K_{1} n^{1+\alpha}$ for some integer $\alpha$, then to prove the theorem we need a control on the derivatives $d^{k} / d \hat{\lambda}^{k}$ up to the order $2+\alpha$. We conjecture that the phase diagram at low temperature is different from the one at zero temperature if the control on derivatives stops to some order less than required by the diophantine properties of $\omega$.

Acknowledgements. F. K and D. P. thank the Swiss National Science Foundation for financial support and M. Z. the Institut de Physique Théorique de l'Université de Lausanne, where this work was done, for warm hospitality.

\section{References}

1. Basuev, A. G.: Complete phase diagrams with respect to external fields at low temperatures for models with nearest-neighbour interaction in the case of a finite or countable number of ground states. Theor. Math. Phys. 58, 171-182 (1984)

2. Bohr, H.: Fastperiodische Funktionen. Berlin: Springer 1932

3. Bricmont, J., Kuroda, K., Lebowitz, J. L.: First order phase transitions in lattice and continuous systems: Extension of Pirogov--Sinai theory. Commun. Math. Phys. 101, 501-538 (1985)

4. Bricmont, J., Slawny, J.: First order phase transitions and perturbation theory. In: Proceedings Groningen (1985), pp. 10-51. Dorlas, T. C., Hugenholtz, N. M., Winnink, M. (eds.). Lecture Notes in Physics Vol. 257. Berlin, Heidelberg, New York: Springer 1986

5. Dinaburg, E. I., Sinai, Ya. G.: An analysis of ANNNI model by Peierl's contour method. Commun. Math. Phys. 98, 119-144 (1984)

6. Dobrushin, R. L., Zahradník, M.: Phase diagrams for continuous spin models: An extension of the Pirogov-Sinai theory. In: Mathematical problem of stat. mechanics and dynamics, pp.1-123. Dobrushin, R. L. (ed.). Dordrecht: D. Reidel 1986

7. Gawędzki, K., Kotecký, R., Kupiainen, A.: Coarse graining approach to first order phase transitions. J. Stat. Phys. 47, 701-724 (1987)

8. Holický, P., Kotecký R., Zahradník, M.: (to appear)

9. Inbrie, J.Z.: Phase diagrams and cluster expansions for the low temperature $P(\phi)_{2}$ models, I and II. Commun. Math. Phys. 82, 261-304 (1981) and 82, 305-343 (1981)

10. Kotecký, R., Laanait, L., Messager, A., Ruiz, J.: The $q$-state Potts model in the standard Pirogov-Sinai theory: Topological aspects. Preprint Marseille CPT-86/P.1948

11. Koukiou, F., Petritis, D., Zahradník, M.: Low temperature phase diagram for models with small quasiperiodic interactions. J. Phys. A: Math. Gen 21 L37-L41 (1988)

12. Malyshev, V. A.: Cluster expansions in lattice models of statistical physics and the quantum theory of fields. Russ. Math. Surv. 35, 1-62 (1980) 
13. Miekisz, J.: Many phases in systems without periodic ground states. Commun. Math. Phys. 107, $577-586(1986)$

14. Moon Park, Y:: Extension of Pirogov-Sinai theory of phase transitions to infinite range of interaction I and II. Commun. Math. Phys. 114, 187-242 (1988)

15. Pirogov, S. A., Sinai, Ya. G.: Phase diagrams of classical lattice systems. Theor. Math. Phys. 25, 1185-1192 (1975), and 26, 39-49 (1976)

16. Radin, Ch.: Crystals and quasi crystals: A continuum model. Commun. Math. Phys. 105, 385-390 (1986)

17. Simon, B.: Almost periodic Schrödinger operators: A review. Adv. Math. 3, 463-490 (1982)

18. Sinai, Ya. G.: Theory of phase transitions: Rigorous results. Oxford: Pergamon Press 1982

19. Slawny, J.: Low temperature properties of classical lattice systems. In: Phase transitions and critical phenomena Vol. 10. Domb, C., Lebowitz, J. L. (eds.) London: Academic Press 1985

20. Zahradník, M.: An alternate of Pirogov-Sinai theory. Commun. Math. Phys. 93, 559-581 (1984)

21. Zahradník, M: Analyticity of low temperature phase diagrams of lattice spin models. J. Stat. Phys. 47, 725-755 (1987)

Communicated by J. Fröhlich

Received October 20, 1987; revised form March 5, 1988 
